\title{
La adaptación de las empresas a la realidad COVID: una revisión sistemática
}

\section{The adaptation of companies to the COVID reality: a systematic review}

Dr. Miguel Ángel García-Madurga es profesor e investigador de la Universidad de Zaragoza (España) (madurga@unizar.es) (https://orcid.org/0000-0002-7816-0050).

Lcda. Ana Julia Grilló-Méndez es profesora e investigadora de la Universidad de Zaragoza (España) (agrillo@unizar.es) (http://orcid.org/0000-0002-6272-5811).

Lcda. Tamara Morte-Nadal es investigadora de la Universidad de Zaragoza (España) (tmorte@unizar.es) (https://orcid.org/0000-0003-4459-9361).

\section{Resumen}

La súbita irrupción de la pandemia COVID-19 ha propiciado profundos cambios sociales y económicos. Las empresas se han visto obligadas a pivotar sus modelos de negocio para asegurar su continuidad. El presente estudio profundiza en las tendencias observadas en los cambios de los modelos de negocio durante esta crisis. El objetivo de esta investigación ha sido identificar los factores que se encuentran detrás de las iniciativas que han adoptado las empresas, pues presumiblemente se consolidarán y serán la base de disrupciones que eran impensables antes de la pandemia. Una rápida revisión sistemática ha permitido recuperar y resumir los resultados de las investigaciones más relevantes en este campo. Se han seleccionado veintiocho artículos de las principales bases de datos científicas, Scopus y Web of Science, utilizando el diagrama de flujo de decisiones de inclusión propuesto por PRISMA (Preferred Reporting Items for Systematic Reviews and Meta-Analysis). Los principales hallazgos del análisis cualitativo que se ha realizado destacan la importancia de la tecnología, con la generalización de canales digitales de marketing y ventas en las empresas, el teletrabajo y el consumo de productos tecnológicos como la Inteligencia Artificial; la adopción de innovaciones relacionadas con la propuesta de valor, en la mayoría de las ocasiones en forma de nuevos productos y servicios, como forma más común de experimentación de cara al consumidor; y la generalización de iniciativas de colaboración entre todos los actores del ecosistema empresarial.

\begin{abstract}
The sudden emergence of the COVID-19 pandemic has led to profound social and economic changes. Companies have been forced to pivot their business models to ensure their continuity. This study delves into the trends observed in the changes in business models during this crisis. The objective of this research has been to identify the factors behind the initiatives they have taken, as they will presumably be consolidated and will be the basis for disruptions unthinkable before the pandemic. A rapid systematic review has allowed to recover and summarize the results of the most relevant research in this field. Twenty-eight articles have been selected from the main scientific databases, Scopus and Web of Science, using the inclusion decision flowchart proposed by PRISMA (Preferred Reporting Items for Systematic Reviews and Meta-Analysis). The main findings of qualitative analysis that has been carried out highlight the importance of technology, with the generalization of digital marketing and sales channels in companies, teleworking and consumption of technological products such as Artificial Intelligence; the adoption of innovations related to the value proposition, in most cases in the form of new products and services, as a more common form of consumer experimentation; and the generalization of collaborative initiatives among all actors in the business ecosystem.
\end{abstract}

\section{Palabras clave I keywords}

Revisión, PRISMA, modelo de negocio, ecosistema empresarial, cooperación entre empresas, innovación, tecnología, COVID-19.

Review, PRISMA, business model, business environment, inter-firm cooperation, innovation, technology, COVID-19.

Cómo citar: García-Madurga, M.A., Grilló-Méndez, A.J., y Morte-Nadal, T. (2021). La adaptación de las empresas a la realidad COVID: una revisión sistemática. Retos Revista de Ciencias de la Administración y Economía, 11(21), pp. 55-70. https://doi.org/10.17163/ret.n21.2021.04 


\section{Introducción}

La repentina aparición del virus COVID-19 ha ocasionado profundos cambios en la sociedad en su conjunto y en el entorno empresarial en particular, en un contexto VUCA (volátil, incierto, complejo y ambiguo) como el que vivimos, que obliga a reconsiderar continuamente rutinas para sobrevivir (García y Esteban, 2020). Es, obviamente, muy pronto para determinar si la pandemia traerá consigo cambios económicos, sociales y políticos permanentes, si bien tendencias como el teletrabajo, y el uso generalizado de Internet parecen haber llegado para quedarse (Tisdell, 2020). Aunque estas tendencias ya estaban en marcha antes de la COVID, se han acelerado, quedando abierta la pregunta de si son o no social y psicológicamente deseables (Tisdell, 2017; Villasmil, 2021).

Con muchos países imponiendo prohibiciones o restricciones a los viajes, la actividad económica se ha debilitado debido a la incertidumbre en el entorno empresarial (Ratten, 2020). Los confinamientos implementados por buen número de países han contribuido, al menos parcialmente, a minimizar la extensión de la pandemia, si bien sus consecuencias tanto psicológicas (Le \& My Nguyen, 2020) como económicas (Acemoglu et al., 2020; Miles et al., 2020; Harris et al., 2020) e, incluso, medioambientales (Helm, 2020) han sido cuantiosas. La gravedad de la recesión se ha debido a los efectos combinados de los shocks tanto del lado de la demanda como de la oferta y requiere replantearse cómo las empresas industriales con cadena de suministro y fabricación global pueden sobrevivir al impacto de situaciones de bloqueo y distanciamiento social. Quizás la pandemia acelerará el proceso de desglobalización y la reducción del comercio y las alianzas de investigación colaborativa entre países y regiones (George et al., 2020).

La desinformación o la información insuficiente o contradictoria han dificultado la capacidad de los consumidores para comprender, planificar y hacer frente a las amenazas sanitarias, económicas y sociales que han provocado cambios en el mercado y en el comportamiento sociocultural de los consumidores (Campbell et al., 2020). Por ejemplo, estudios recientes sobre patrones de gasto de los consumidores proporcionan evidencia de desplazamiento espacial y temporal del consumo y comportamientos de almacenamiento (Hall et al., 2021), pues el comportamiento del consumidor y los factores personales, sociales, psicológicos y culturales se encuentran asociadas significativamente (Ortega-Vivanco, 2020). En tanto en cuanto la situación se normalice y los consumidores retomen los viejos hábitos, es plausible pensar que estos serán modificados por nuevas regulaciones y procedimientos. También surgirán nuevos hábitos por los avances tecnológicos, los cambios demográficos y las formas innovadoras en que los consumidores han aprendido a lidiar con la difuminación de los límites del trabajo, el ocio y la educación (Sheth, 2020a).

Las crisis provocan turbulencias en el mercado debido a cambios en las rutinas y estructuras (Williams et al., 2017), si bien su impacto es muy desigual en función de los sectores. Respecto a lo que está aconteciendo como consecuencia de la pandemia, en algunos sectores, como la educación, se suceden las innovaciones; en otros, como la hostelería y el turismo, situadas en el epicentro de la pandemia, el panorama parece sombrío. Empresas como Amazon o Wallmart salen reforzadas, mientras muchas otras se han visto obligadas a cerrar sus puertas (Barrero et al., 2020). En particular, las PYMES, por su particular idiosincrasia, se han visto impactadas dramáticamente y requieren de decisiones institucionales, estratégicas y financieras muy específicas (Eggers, 2020), así como de la digitalización y el aprovechamiento del canal online, tras un cuidadoso análisis de la temporalidad de las ventanas de oportunidades y la vulnerabilidad de las innovaciones que se requieren (Dannenberg et al., 2020).

Los trabajadores más jóvenes, las mujeres y las minorías étnicas se han visto afectados de manera desproporcionada y han visto peligrar sus empleos. Los departamen- 
tos de Recursos Humanos tienen la oportunidad de desempeñar un papel importante ayudando a las personas y las organizaciones a encontrar y apoyar la resiliencia, gestionar las crisis y construir culturas profesionales más sostenibles (Hite \& McDonald, 2020); de hecho, la crisis sanitaria ha trasladado al presente la conversación sobre el futuro del trabajo (Lund et al., 2020). Siguiendo a Hongwei y Lloyd (2020), la pandemia ofrece también una gran oportunidad para que las empresas caminen hacia una Responsabilidad Social Empresarial (RSE) más genuina y auténtica y contribuyan a abordar los urgentes desafíos sociales y ambientales.

En este marco, las empresas deben convertirse en mejores clientes para sus proveedores y redefinir el propósito de sus negocios (Sheth, 2020b), y los decisores revisar sus modelos comerciales, centrándose en los clientes, las propuestas de valor, las capacidades, el potencial de colaborar con organizaciones a priori competidoras y las conexiones entre todos estos temas (Crick \& Crick, 2020). Y eso, rápidamente, considerando los efectos futuros de estas decisiones y, simultáneamente, generando opciones para superar la crisis aquí y ahora (Teece et al., 2016) y reflexionando sobre los factores clave en la identificación de nuevas oportunidades (Gamero \& Ostos, 2020).

Conforme la pandemia ha ido avanzando, las organizaciones han ido integrando total o parcialmente estas recomendaciones y reajustando sus enfoques, actividades y modelos de negocio, esto es, la forma en la que crean y entregan valor a sus clientes (Seddon et al., 2004). La revisión realizada profundiza en las tendencias observadas en los cambios en los modelos de negocio durante el año 2020. Las revisiones sistemáticas rápidas surgen como una metodología útil para proporcionar evidencias procesables y relevantes de modo eficiente cuando los factores tiempo y/o coste son relevantes (Tricco et al., 2015), permiten examinar áreas emergentes (Trico et al., 2016) y son útiles para responder preguntas de investigación como ¿qué información se ha presentado sobre este tema en la literatura? (Sucharew \& Macaluso, 2019). Las revisiones sistemáticas y otras técnicas empleadas para la síntesis de conocimiento son cada vez más empleadas para facilitar la toma de decisiones (Bosch-Capblanch et al., 2012).

En situaciones donde se requieren decisiones urgentes, los gobiernos, las partes interesadas en el sistema de salud, las organizaciones internacionales y la sociedad civil solicitan revisiones rápidas (Tricco et al., 2017). Se han publicado en una variedad de áreas incluida la comunicación digital, el consumo de bebidas energéticas por parte de los niños, las intervenciones de salud sexual y el abandono del cannabis (Plüddemann et al., 2018). Estas investigaciones respetan los principios clave de las revisiones sistemáticas, incluida una declaración clara de los objetivos, la predefinición de los criterios de elegibilidad, la evaluación de la validez de los resultados y la presentación sistemática de los resultados (Langlois et al., 2017), con la salvedad de que los procesos se aceleran y optimizan para completar su realización en un plazo inferior. Las revisiones rápidas son rigurosas y explícitas en el método y, por lo tanto, sistemáticas, pero hacen concesiones a la amplitud o profundidad del proceso de revisión al limitar algunos aspectos. Para ello, se identifican varias técnicas válidas que pueden usarse para acortar el tiempo, como enfocar cuidadosamente la pregunta, usar estrategias de búsqueda menos sofisticadas, restringir la cantidad de literatura gris y/o extraer solo variables clave (Grant \& Booth, 2009). Este tipo de estudios involucran menos partes interesadas, menos discusiones, menos iteraciones y un mayor uso de anteriores lecciones aprendidas (Oliver et al., 2017); su elaboración es un reto importante, por la dificultad de responder en corto plazo, con rigor científico y un estándar de transparencia aceptable por todos los interesados (Watt et al., 2008).

La crisis ha puesto encima de la mesa temas de investigación tan dispares como su impacto en sectores específicos como el turístico (Sigala, 2020); la identificación de 
diferentes tipos de incertidumbre, sus antecedentes y resultados, y las estrategias utilizadas para mitigar su impacto (Sharma et al., 2020); el liderazgo eficaz en entornos de trabajo virtuales (Bartsch et al., 2021); las consecuencias a largo plazo de la pandemia en el diseño de las organizaciones (Foss, 2020); la prestación de servicios esenciales, las prácticas de compra responsable, el comportamiento del mercado en medio de la crisis (Mele et al., 2020); o las necesidades que generarán eventuales modelos híbridos, presenciales y remotos, de trabajo (Shankar, 2020).

El objetivo de nuestro estudio es analizar cómo han respondido las empresas a la pandemia COVID-19, caracterizada, como se ha descrito, por dificultades operacionales de todo tipo y cambios radicales en los hábitos de compra de los consumidores. A partir del análisis de los documentos seleccionados, se dilucidará esta pregunta de investigación: ¿qué grandes tendencias guiarán los pasos de las organizaciones empresariales tras la crisis?

El manuscrito se estructura de esta manera: identificación del problema de investigación a partir de la revisión de la literatura (Sección 1); explicación detallada de la metodología utilizada (Sección 2); presentación de los resultados (Sección 3); y, finalmente, discusión basada en los resultados del estudio y sus limitaciones e implicaciones para estudios futuros (Sección 4).

\section{Criterios de selección del corpus}

La investigación se ha realizado de acuerdo con el protocolo Preferred Reporting Items for Systematic Reviews and Meta-Analyses PRISMA (Moher et al., 2009). PRISMA fue elaborado por un grupo de expertos que identificaron los criterios mínimos para revisiones sistemáticas destinadas a publicaciones científicas de alta calidad. Todavía no existe una extensión de PRISMA para revisiones rápidas, si bien el empleo de la lista de verificación PRISMA aumenta la transparencia de aquellas (Kelly et al., 2016) y facilita la trazabilidad de todo el proceso en general y del flujo de la información en particular. Siguiendo las recomendaciones de Hartling et al. (2015), y alineados con la propuesta de PRISMA, la investigación se caracterizó por su transparencia y la claridad de su propósito.

Se siguió un proceso de cuatro etapas a partir del objeto de investigación: identificación de estudios relevantes, selección de estudios, cartografía de los datos y síntesis e informe de los resultados.

En primer lugar, se especificaron y documentaron los criterios de inclusión y exclusión, tal y como se muestran en la Tabla 1. Se pretendió identificar propuestas con base en estudios científicos, pese a que estos aún son escasos en número por encontrarnos todavía inmersos en la pandemia. Puesto que la difusión en la comunidad científica de las actas de congresos y el factor de impacto de los libros y capítulos son menores que los de los artículos científicos, ambas fuentes fueron excluidas.

\section{Tabla 1. Criterios de inclusión y de exclusión}

\section{Criterios de inclusión}

Criterios de exclusión

Artículos de investigación publicados (o en prensa) en revistas científicas.

Publicados en 2020-2021.

Escritos en inglés.

Libros y capítulos de libros.

Área de Business Management o Social Sciences.

Fuente: Elaboración propia. 
Se realizó a continuación una búsqueda exhaustiva en las bases de datos Scopus (www.scopus.com) y Web of Science (https://apps.webofknowledge.com/). Para construir la ecuación de búsqueda óptima, se utilizaron en SCOPUS el día 19 de diciembre combinaciones de palabras clave y frases relacionadas con los negocios y la COVID. La ecuación de búsqueda finalmente utilizada, incluyendo limitadores para tener en cuenta los criterios de inclusión y de exclusión, fue: TITLE-ABS-KEY (business AND covid) AND (LIMIT-TO (DOCTYPE, “ar") OR LIMIT-TO (DOCTYPE, "re")) AND (LIMIT-TO (SRCTYPE, “j”)) AND (LIMIT-TO (SUBJAREA, “SOCI”) OR LIMIT-TO (SUBJAREA, "BUSI") OR LIMIT-TO (SUBJAREA, "MULT")). La búsqueda con ayuda de esta ecuación y su análoga en Web of Science resultó en 882 documentos. Los resultados se exportaron a EndNote, y se eliminaron los duplicados. La detección y eliminación de estudios duplicados eliminó 91 citas, quedando finalmente 791 artículos.

Se realizó más adelante un examen de evidencia con base en el título y el resumen. Se excluyeron 688 estudios al no ajustarse al objetivo de la investigación desde la perspectiva del alcance del estudio. Luego, se realizó una evaluación del grado de interés de los 103 documentos escogidos, seleccionando aquellas aportaciones transversales y/o generalizables a modelos de negocio en un sentido amplio, y excluyendo aquellos resultados específicos de productos o zonas geográficas. En conclusión, se seleccionaron 28 documentos para la síntesis cualitativa. La Figura 1 muestra el diagrama de flujo de decisión de inclusión con los pasos del proceso de decisión de revisión (identificación, selección, elegibilidad e inclusión) de acuerdo con el estándar PRISMA:

\section{Figura 1. Diagrama de flujo de las decisiones de inclusión}

\begin{tabular}{|c|c|c|}
\hline $\begin{array}{l}\text { Registros identificados en bases de datos } \\
\qquad(n=882)\end{array}$ & $\rightarrow$ & $\begin{array}{l}\text { Duplicados eliminados } \\
\qquad(n=91)\end{array}$ \\
\hline \multicolumn{3}{|l|}{$\downarrow$} \\
\hline $\begin{array}{l}\text { Registros para screening } \\
\qquad(n=791)\end{array}$ & $\rightarrow$ & $\begin{array}{l}\text { Registros excluidos por estar } \\
\text { fuera del alcance } \\
(n=688)\end{array}$ \\
\hline$\downarrow$ & \multirow[b]{2}{*}{$\rightarrow$} & \multirow[b]{2}{*}{$\begin{array}{l}\text { Registros excluidos por su especificidad } \\
\qquad(n=75)\end{array}$} \\
\hline $\begin{array}{l}\text { Registros seleccionados por } \\
\text { título y resumen } \\
(n=103)\end{array}$ & & \\
\hline$\downarrow$ & & \\
\hline $\begin{array}{l}\text { Estudios incluidos en la síntesis cualitativa } \\
\qquad(n=28)\end{array}$ & & \\
\hline
\end{tabular}

Elaboración propia a partir de Preferred Reporting Items for Systematic Reviews and Meta-Analyses (PRISMA).

Los estudios incluidos en la síntesis se recogen en la Tabla 2 con todos los datos relevantes para informar el objetivo de revisión del alcance. Los campos de extracción fueron: título, primer autor, revista y aportación principal. 


\section{Tabla 2. Documentos incluidos en la síntesis cualitativa}

\begin{tabular}{|c|c|c|c|}
\hline Título & Primer autor & Revista & Aportación principal \\
\hline $\begin{array}{l}\text { COVID-19 impact and survi- } \\
\text { val strategy in business tou- } \\
\text { rism market: the example of } \\
\text { the UAE MICE industry }\end{array}$ & $\begin{array}{l}\text { Aburumman, A. } \\
\text { A. }\end{array}$ & $\begin{array}{l}\text { Humanities and } \\
\text { Social Sciences } \\
\text { Communications }\end{array}$ & $\begin{array}{l}\text { Importancia de la exter- } \\
\text { nalización como fuente de } \\
\text { variabilización de costes }\end{array}$ \\
\hline $\begin{array}{l}\text { Cutting-edge technologies } \\
\text { for small business and inno- } \\
\text { vation in the era of COVID-19 } \\
\text { global health pandemic }\end{array}$ & Akpan, I. J. & $\begin{array}{l}\text { Journal of Small } \\
\text { Business } \mathbb{E} \\
\text { Entrepreneurship }\end{array}$ & $\begin{array}{l}\text { Dificultades de adaptación } \\
\text { de las PYMES al nuevo es- } \\
\text { cenario por su cultura con- } \\
\text { servadora en lo relativo a } \\
\text { los cambios tecnológicos }\end{array}$ \\
\hline $\begin{array}{l}\text { Small business awareness } \\
\text { and adoption of state-of-the- } \\
\text { art technologies in emerging } \\
\text { and developing markets, and } \\
\text { lessons from the COVID-19 } \\
\text { pandemic }\end{array}$ & Akpan, I.J. & $\begin{array}{l}\text { Journal of Small } \\
\text { Business } \mathcal{E} \\
\text { Entrepreneurship }\end{array}$ & $\begin{array}{l}\text { Ventajas competitivas } \\
\text { aportadas por la implanta- } \\
\text { ción de tecnología de van- } \\
\text { guardia }\end{array}$ \\
\hline $\begin{array}{l}\text { The Challenges and Oppor- } \\
\text { tunities in the Digitalization } \\
\text { of Companies in a Post-CO- } \\
\text { VID-19 World }\end{array}$ & Almeida, F. & $\begin{array}{l}\text { IEEE Engineering } \\
\text { Management Review }\end{array}$ & $\begin{array}{l}\text { Impacto de los procesos de } \\
\text { transformación digital so- } \\
\text { bre diferentes áreas de los } \\
\text { negocios }\end{array}$ \\
\hline $\begin{array}{l}\text { Expanding entrepreneurial } \\
\text { solution spaces in times of } \\
\text { crisis: Business model experi- } \\
\text { mentation amongst packaged } \\
\text { food and beverage ventures }\end{array}$ & Björklund, T.A. & $\begin{array}{l}\text { Journal of Business } \\
\text { Venturing Insights }\end{array}$ & $\begin{array}{l}\text { Importancia de lo colabo- } \\
\text { rativo en momentos de cri- } \\
\text { sis }\end{array}$ \\
\hline $\begin{array}{l}\text { The Future of B2B Custo- } \\
\text { mer Solutions in a Post-CO- } \\
\text { VID-19 Economy: Manage- } \\
\text { rial Issues and an Agenda for } \\
\text { Academic Inquiry }\end{array}$ & Bond III, E.U. & $\begin{array}{l}\text { Journal of Service } \\
\text { Research }\end{array}$ & $\begin{array}{l}\text { Adaptación de las empre- } \\
\text { sas de servicios en el entor- } \\
\text { no B2B a la nueva realidad }\end{array}$ \\
\hline $\begin{array}{l}\text { The role of business model } \\
\text { innovation in the hospitali- } \\
\text { ty industry during the CO- } \\
\text { VID- } 19 \text { crisis }\end{array}$ & Breier, M. & $\begin{array}{l}\text { International Journal } \\
\text { of Hospitality } \\
\text { Management }\end{array}$ & $\begin{array}{l}\text { Innovaciones incrementa- } \\
\text { les y de rápida implanta- } \\
\text { ción como respuesta a la } \\
\text { crisis }\end{array}$ \\
\hline $\begin{array}{l}\text { Will COVID- } 19 \text { be the tip- } \\
\text { ping point for the Intelligent } \\
\text { Automation of work? A re- } \\
\text { view of the debate and impli- } \\
\text { cations for research }\end{array}$ & Coombs, C. & $\begin{array}{l}\text { International journal } \\
\text { of information } \\
\text { management }\end{array}$ & $\begin{array}{l}\text { La automatización y la im- } \\
\text { plantación de soluciones } \\
\text { de Inteligencia Artificial en } \\
\text { el nuevo escenario empre- } \\
\text { sarial }\end{array}$ \\
\hline $\begin{array}{l}\text { Coopetition and COVID-19: } \\
\text { Collaborative business-to-bu- } \\
\text { siness marketing strategies in } \\
\text { a pandemic crisis }\end{array}$ & Crick, J.M. & $\begin{array}{l}\text { Industrial Marketing } \\
\text { Management }\end{array}$ & $\begin{array}{l}\text { Pervivencia de los modelos } \\
\text { colaborativos una vez la } \\
\text { crisis esté definitivamente } \\
\text { controlada }\end{array}$ \\
\hline $\begin{array}{l}\text { Artificial intelligence in the } \\
\text { agri-food system: Rethinking } \\
\text { sustainable business models } \\
\text { in the COVID-19 scenario }\end{array}$ & Di Vaio, A. & Sustainability & $\begin{array}{l}\text { La Inteligencia Artificial } \\
\text { en la creación y desarrollo } \\
\text { de modelos de negocio sos- } \\
\text { tenibles }\end{array}$ \\
\hline
\end{tabular}


Título

Primer autor

Impact of COVID-19 pandemic on information management research and practice: Transforming education, work and life

The strategic change matrix and business sustainability across COVID-19

Reframing service innovation: COVID-19 as a catalyst for imposed service innovation

Opportunities of frugality in
the post-corona era

the post-corona era

Herstatt, C.

Business model innovation
in established SMEs: A con-
in established SMEs: A configurational approach

The triple-edged sword of COVID-19: understanding the use of digital technologies and the impact of productive, disruptive, and destructive nature of the pandemic

Business-to-business marketing responses to COVID-19 crisis: a business process perspective

New technologies used in COVID-19 for business survival: Insights from the Hotel Sector in China

Pivoting to stay the course: How women entrepreneurs take advantage of opportunities created by the COVID-19 pandemic

Kamal, M.M.

Kang, J.
Revista Aportación principal

International Journal logía de gestión de la inof Information formación y sus ramifica-

Management $\quad$ ciones en el entorno de la pandemia

La Inteligencia de Mercado como herramienta de competitividad en un marco complejo

Vectores fuerza en el ámbito de las decisiones innovadoras adoptadas durante la crisis

La frugalidad como megatendencia que puede dar forma a una frugal "excelencia verde asequible" como el paradigma dominante de la innovación

Medidas para asegurar la exitosa implantación de innovaciones en modelos de negocio de PYMES

Uso de tecnologías digitales avanzadas para evitar la pérdida de productividad, la interrupción en las operaciones del negocio y la destrucción de empresas

Information Systems

Management

Innovation:

Technology, Market, and Complexity

Management

(Mc

Marketing

Intelligence $\mathbb{E}$

Planning

Respuestas a la crisis desde el marketing

Tecnologías específicas para el tratamiento de las nuevas formas de relación provocadas por la pandemia

Pivotes del modelo de negocio en las empresas propiedad de mujeres para, simultáneamente, reducir el riesgo y aprovechar nuevas oportunidades 


\begin{tabular}{|c|c|c|c|}
\hline Título & Primer autor & Revista & Aportación principal \\
\hline $\begin{array}{l}\text { New and novel business pa- } \\
\text { radigms in and from China } \\
\text { and India }\end{array}$ & Paul, J. & $\begin{array}{l}\text { European Business } \\
\text { Review }\end{array}$ & $\begin{array}{l}\text { Impacto geopolítico de de- } \\
\text { cisiones adoptadas por las } \\
\text { empresas de los dos gran- } \\
\text { des manufactureros a nivel } \\
\text { mundial }\end{array}$ \\
\hline $\begin{array}{l}\text { Resilience measures to dea- } \\
\text { ling with the COVID-19 } \\
\text { pandemic. Evidence from } \\
\text { Romanian micro and small } \\
\text { enterprises }\end{array}$ & PØunescu, C & $\begin{array}{l}\text { Management } \\
\mathcal{E} \text { Marketing. } \\
\text { Challenges for the } \\
\text { Knowledge Society }\end{array}$ & $\begin{array}{l}\text { Temores empresariales } \\
\text { frente a la crisis y su im- } \\
\text { pacto en la toma de deci- } \\
\text { siones }\end{array}$ \\
\hline $\begin{array}{l}\text { COVID-19: A Huge Opportu- } \\
\text { nity for Innovation in Marke- } \\
\text { ting }\end{array}$ & Pillania, R.K. & $\begin{array}{l}\text { Indian Journal of } \\
\text { Marketing }\end{array}$ & $\begin{array}{l}\text { La mayor percepción del } \\
\text { riesgo ha creado oportuni- } \\
\text { dades para las innovacio- } \\
\text { nes de marketing }\end{array}$ \\
\hline $\begin{array}{l}\text { Exploring the relationship } \\
\text { between social capital, inno- } \\
\text { vation capability and innova- } \\
\text { tion during the coronavirus } \\
\text { pandemic }\end{array}$ & Putra, I. & $\begin{array}{l}\text { Uncertain Supply } \\
\text { Chain Management, }\end{array}$ & $\begin{array}{l}\text { La innovación y su víncu- } \\
\text { lo con el éxito empresarial } \\
\text { durante la pandemia }\end{array}$ \\
\hline $\begin{array}{l}\text { Navigating disruptive crises } \\
\text { through service-led growth: } \\
\text { The impact of COVID-19 on } \\
\text { Italian manufacturing firms }\end{array}$ & Rapaccini, M. & $\begin{array}{l}\text { Industrial Marketing } \\
\text { Management }\end{array}$ & $\begin{array}{l}\text { Importancia de los mode- } \\
\text { los de negocio de servitiza- } \\
\text { ción, de la aceleración de } \\
\text { la transformación digital y } \\
\text { de los servicios avanzados } \\
\text { en el contexto de la pande- } \\
\text { mia }\end{array}$ \\
\hline $\begin{array}{l}\text { Sustainability learnings from } \\
\text { the COVID-19 crisis. Oppor- } \\
\text { tunities for resilient industry } \\
\text { and business development }\end{array}$ & Schaltegger, S. & $\begin{array}{l}\text { Sustainability } \\
\text { Accounting, } \\
\text { Management and } \\
\text { Policy Journal. }\end{array}$ & $\begin{array}{l}\text { Aprendizajes para conse- } \\
\text { guir negocios más resilien- } \\
\text { tes y sostenibles en entor- } \\
\text { nos de crisis }\end{array}$ \\
\hline $\begin{array}{l}\text { Business models shifts: Im- } \\
\text { pact of Covid-19 }\end{array}$ & Seetharaman, $\mathrm{P}$. & $\begin{array}{l}\text { International Journal } \\
\text { of Information } \\
\text { Management }\end{array}$ & $\begin{array}{l}\text { Rediseño de productos y } \\
\text { modelos de negocio para } \\
\text { el éxito en el nuevo ecosis- } \\
\text { tema }\end{array}$ \\
\hline $\begin{array}{l}\text { Business of business is more } \\
\text { than business: Managing du- } \\
\text { ring the Covid crisis }\end{array}$ & Sheth, J. & $\begin{array}{l}\text { Industrial Marketing } \\
\text { Management }\end{array}$ & $\begin{array}{l}\text { Implicaciones de la crisis } \\
\text { en los negocios B2B }\end{array}$ \\
\hline $\begin{array}{l}\text { A systematic review and fu- } \\
\text { ture directions of the sharing } \\
\text { economy: business models, } \\
\text { operational insights and en- } \\
\text { vironment-based utilities }\end{array}$ & Zhu, X. & $\begin{array}{l}\text { Journal of Cleaner } \\
\text { Production }\end{array}$ & $\begin{array}{l}\text { Oportunidades de los mo- } \\
\text { delos colaborativos en el } \\
\text { nuevo escenario COVID }\end{array}$ \\
\hline
\end{tabular}

Fuente: Elaboración propia.

\section{Debates y aportes al estado de la cuestión}

Las crisis son catalizadoras de tendencias (Bond III et al., 2020), pues llevan ineludiblemente a la experimentación, introducen mecanismos novedosos de gestión 
de riesgos, exigen el redespliegue de determinados recursos y aceleran la adopción de herramientas (en el caso estudiado, las digitales). La súbita irrupción de la pandemia ha requerido de las empresas la adopción de decisiones de toda índole. Sectores como el MICE (Meetings, Incentives, Conferences and Exhibitions) han recurrido a medidas operacionales obvias, como recurrir a la subcontratación, buscando la reducción de costes operativos (Aburumman, 2020). Kang et al. (2020) han identificado nueve respuestas urgentes desde el marketing a la crisis COVID; ajustar los productos de forma proactiva para las necesidades emergentes; coordinar proveedores para satisfacer la creciente demanda; migrar a canales de distribución digitales; promover la solidaridad con la totalidad de los miembros de la cadena de suministro; invertir en publicidad y promoción; fomentar la venta cruzada; y apoyar a los clientes existentes.

Superado el primer y severo golpe, empresas de todos los sectores se han replanteado su sostenibilidad: las investigaciones de Păunescu y Mátyus (2020) revelan cómo sus principales temores el mantenimiento del negocio desde el punto de vista de la sostenibilidad financiera, la inseguridad económica y la alta probabilidad de pérdidas de empleo. Las denominadas 3C (competencias, capacidades y competitividad) ofrecen un marco interno capaz de hacer avanzar a la empresa hacia ese futuro asegurado (Hamilton, 2020). La "nueva normalidad" debe basarse en un enfoque conciliatorio que asegure un acceso asequible a los productos y servicios para la población, gestione adecuadamente el consumo de la sociedad de recursos naturales y luche por la excelencia tecnológica (Herstatt \& Tiwari, 2020). Se trata, además, de generar aprendizajes para conseguir negocios más resilientes y sostenibles en entornos de pandemia (Schaltegger, 2020) y de orientarse sistemáticamente a las partes interesadas, midiendo la satisfacción y el bienestar de la comunidad, los proveedores, los clientes y los empleados (Sheth, 2020b).

La revisión sistemática realizada permite englobar las iniciativas adoptadas por las empresas para adaptarse a la compleja situación generada por la pandemia en torno a tres grandes vectores: la innovación, la tecnología y la colaboración.

\subsection{La innovación}

Si bien la necesidad de generar resultados en un entorno de tan alta incertidumbre ha supuesto una extraordinaria presión para las personas responsables de la toma de decisiones, los confinamientos y la disminución del número de actividades operativas les han liberado tiempo y permitido reflexionar a nivel estratégico y, en particular, analizar cómo la innovación, en sus distintas expresiones, puede contribuir al futuro sostenible de sus organizaciones.

Innovaciones relacionadas con la propuesta de valor han sido la forma más común de experimentación de cara al consumidor, adoptando en la mayoría de las ocasiones la forma de nuevos productos y servicios. La crisis de la COVID-19 ha requerido, en el corto plazo, que las organizaciones buscaran opciones digitales e identificaran formas de entregar sus productos y servicios con un contacto físico mínimo y seguro, así como posiciones estratégicas y socios en el nuevo ecosistema que pudieran ayudarlas a lograrlo. Para lograr esta adaptación a los tiempos cambiantes, las empresas han debido mostrarse tremendamente ágiles; explotar sus capacidades dinámicas (Seetharaman, 2020); exprimir las oportunidades que les ha brindado el marketing, tales como agrupar productos, adoptar iniciativas de rebranding e, incluso, ingresar en categorías de productos completamente nuevas (Björklund et al., 2020); y aprovechar su capital social para crear y ampliar el conocimiento sobre clientes, materias primas y competidores y para obtener información barata, rápida y útil para generar innovación (Putra et al., 2020). 
En sectores seriamente afectados como la hostelería, la innovación en el modelo de negocio se ha producido mediante pequeños cambios incrementales de rápida implementación (Breier et al., 2021). En el sector servicios, la pandemia ha obligado a las empresas a ir más allá de las estrategias comerciales existentes: las innovaciones en este sector se han caracterizado por la flexibilidad espacial, el alcance social, su foco en la salud y la explotación de la tecnología, actuando como motores del cambio los clientes y su nuevo rol, las restricciones externas y la dinámica de las instituciones (Heinonen \& Strandvik, 2020).

No solamente el sector es una variable relevante a estos efectos: el género (Manolova et al., 2020) el país o el tamaño también importan. Así, la concentración de recursos por parte del Gobierno ayuda a crear un entorno empresarial externo que promueve que las empresas se enfoquen en la innovación y el avance tecnológico adecuados (Paul et al., 2020). Respecto al tamaño, más allá de la incertidumbre y la complejidad que enfrentan las PYMES (Pequeñas y Medianas Empresas) al innovar en sus modelos de negocio, los resultados de la investigación de Ibarra et al. (2020) sugieren que este tipo de empresas deben implementar un conjunto específico de capacidades, promover prácticas y rutinas enfocadas en detectar las necesidades del cliente, detectar opciones tecnológicas, conceptualizar y experimentar, colaborar y promulgar una estrategia clara de innovación de sus modelos de negocio. Contrariamente a la percepción general, en lugar de dejar de financiar innovaciones durante tiempos de crisis, las grandes empresas han dedicado esfuerzos y fondos a generar innovaciones durante estos tiempos de crisis, utilizando la COVID-19 como terapia de choque para vencer resistencias internas frente a la innovación (Pillania, 2020).

\subsection{La tecnología}

En tiempos COVID, la tecnología es esencial para mejorar la ventaja competitiva y asegurar la supervivencia. En el competitivo panorama empresarial contemporáneo, pequeñas empresas con tecnología de vanguardia pueden crear y mantener estrategias competitivas y preparar el escenario para el crecimiento a largo plazo y el liderazgo del mercado, diferenciándose de las otras muchas que se resisten a la adopción de estas soluciones y se limitan al uso regular de la infraestructura de tecnología de la información comúnmente utilizada (Akpan et al., 2020a).

La pandemia ofrece una oportunidad para el surgimiento de una nueva generación de empresarios dispuestos a liderar la próxima revolución industrial e inventar nuevas formas de hacer negocios utilizando tecnología punta (Akpan et al., 2020b). Sectores gravemente afectados por la pandemia, como la restauración y la hostelería, pueden mejorar la higiene, promover el distanciamiento social y brindar a sus clientes una experiencia convincente y personalizada gracias al uso de nuevas tecnologías (Lau, 2020).

La COVID-19 ha acelerado los procesos de transformación digital no solo en empresas sino también en particulares y entidades públicas. La digitalización de las empresas aumentará la importancia que se le da a los canales digitales de marketing y ventas en las empresas y fomentará el teletrabajo y el consumo de productos tecnológicos (crecimiento de sistemas compatibles con el Internet de las Cosas; Inteligencia Artificial; Big Data; robótica). Más personas interactuarán utilizando mecanismos de comunicación híbridos accesibles desde cualquier lugar y no exclusivamente en el entorno físico de las empresas y sus hogares. La ciberseguridad y la privacidad aparecen como elementos clave en la adopción de estas nuevas soluciones tecnológicas (Almeida et al., 2020).

Como se adelantó en la introducción, las implicaciones de esta aceleración son numerosas. Las tecnologías digitales pueden ayudar a crear modelos de negocio soste- 
nibles, aumentando la productividad, reduciendo los costos de producción y las emisiones y disminuyendo la intensidad de los recursos del proceso de producción (Di Vaio et al., 2020). Por ejemplo, existe consenso sobre el hecho de que las implementaciones de Inteligencia Artificial (IA) pueden proporcionar valor a las empresas. Sin embargo, en primer lugar, es probable que se centren en tareas que son altamente predecibles y rutinarias. En segundo lugar, se prevé que los niveles de ansiedad en la fuerza laboral por la automatización sean altos debido a la recesión global y los temores sobre la seguridad de los empleos. En tercer lugar, las experiencias de implementación de IA sugieren que las mejoras en el rendimiento de los procesos de negocio a menudo se logran combinando las capacidades técnicas con las habilidades sociales de los trabajadores humanos en equipos de trabajadores híbridos (Coombs, 2020).

En particular, es necesario un cambio de paradigma de $360^{\circ}$ en el sector industrial, estimulando y desarrollando la próxima generación de organizaciones, industrias y sociedades. La clave está en co-trabajar y co-crear para generar datos en tiempo real, reconsiderar la reunión de personas y máquinas y crear plataformas para la creación conjunta de valor, reforzar las áreas de sistemas y tecnologías de la información y, por último, desarrollar infraestructuras digitales para gestionar comunicaciones e interacciones seguras en línea (Kamal, 2020). Las empresas industriales que aprovechen servicios basados en software y/o habilitados digitalmente se verán a futuro menos afectadas por este tipo de crisis (Rapaccini et al., 2020).

La pandemia ha obligado a los gobiernos y a los responsables de la toma de decisiones a reevaluar cómo utilizan la información y extender el uso de la tecnología para mitigar muchos de los impactos sociales de la propagación del virus (Dwivedi et al., 2020). El enorme desafío para los gerentes es involucrarse en este cambio, mientras se intenta mantener el negocio en funcionamiento, frente a un futuro diferente e incierto (Almeida et al., 2020).

\subsection{La colaboración}

En los campos del alojamiento, el transporte, los servicios educativos, la vivienda y el turismo, han surgido durante los últimos años excelentes empresas de economía colaborativa (Zhu \& Liu, 2020). De acuerdo con la investigación de Björklund et al. (2020), durante la crisis los emprendedores han utilizado sus redes para la colaboración y la acción colectiva, lanzando nuevos productos y servicios a través de canales de ventas colaborativos, compartiendo información vital y ayudando simultáneamente a terceros en sus comunidades locales. Estas acciones han sido normalmente incrementales y han contribuido al desarrollo de capacidades internas y de las capacidades relacionales y de los ecosistemas, ampliando aún más su resiliencia y el potencial para la futura creación de valor conjunto.

La cooperación entre empresas ha demostrado ser una estrategia de marketing B2B eficaz en una pandemia, ya que tiene el potencial de beneficiar a las organizaciones, a sus clientes y a otras partes interesadas. Las actividades de cooperación se componen de fuerzas cooperativas y competitivas, por lo que los tomadores de decisiones eligen con extrema precaución los competidores con los que colaboran, para evitar resultados perjudiciales. Una vez finalice la pandemia de la COVID-19, existen dudas sobre si estas estrategias de competencia existentes continuarán o finalizarán (Crick \& Crick, 2020).

\section{Conclusiones y discusión}

La forma en la que las empresas pivotan sus modelos de negocio no puede entenderse completamente sin tener en cuenta la estructura económica y social en la que 
operan. Para que estos movimientos resulten exitosos en tiempos de crisis deben simultáneamente reducir el riesgo y aprovechar las oportunidades (Manilova et al., 2020). Las empresas se han encontrado frente a la compleja tarea de gestionar simultáneamente lo que está cerca, las dificultades financieras y operacionales provocadas por el shock de oferta y demanda, y lo que está lejos, el incierto porvenir que está por llegar. El análisis de los datos obtenidos mediante la técnica empleada ha permitido conocer los factores que han hecho evolucionar los modelos de negocio para adaptarse a la crisis originada por la COVID-19. En primer lugar, las empresas han hecho evolucionar su portafolio de productos y servicios para adaptarlo a las características de una nueva realidad plagada de restricciones como, fundamentalmente, la proximidad física. En segundo lugar, la tecnología ha pasado de ser un futurible a convertirse en una realidad, que debe soportar procesos de manufactura más eficientes, el auge del comercio online, el teletrabajo y la digitalización de todos los procesos de las organizaciones. Por último, la apuesta por enfoques colaborativos, ya consolidados en algunos sectores $\mathrm{y}$ alineados con los principios de la responsabilidad social, ha permitido encauzar la solidaridad de todo el ecosistema empresarial, consciente de la necesidad de la ayuda mutua en momentos de crisis.

El hecho de que en este momento la pandemia siga activa ha implicado una selección y revisión limitadas de la literatura, todavía escasa. Para evitar que el producto final de esta investigación sea confundido con una revisión sistemática estándar se han esbozado explícitamente en la sección de metodología las compensaciones y las limitaciones de un producto rápido en comparación con una revisión sistemática. Reducir la duración del proceso de revisión implica un mayor riesgo de introducir sesgos: documentar la metodología y resaltar sus limitaciones es una forma de luchar contra tales prejuicios (Grant \& Booth, 2009). "Más corto y más rápido" puede no ser siempre mejor, o incluso tan bueno, pero la información disponible actualmente no permite concluir cuándo preocuparse de que las conclusiones de una revisión rápida puedan estar equivocadas (Hartling et al., 2015). Además, las revisiones sistemáticas tienen sus propias limitaciones, sobre las cuales no reflexionamos aquí, y estas limitaciones, por lo tanto, también se aplican a las revisiones rápidas (Møller, 2018).

Nos encontramos como sociedad, desgraciadamente, inmersos en duros momentos y con incertidumbres sobre la evolución de la pandemia. Las tendencias descritas se consolidarán, o no, en función del escenario que finalmente se presente. Futuros trabajos de investigación deberán profundizar en su impacto y en el vínculo entre ellas y las disrupciones que, sin duda, acabarán provocando.

\section{Referencias}

Aburumman, A.A. (2020). COVID-19 impact and survival strategy in business tourism market: the example of the UAE MICE industry. Humanities and Social Sciences Communications, 7(1), 1-11. https://doi.org/10.1057/s41599-020-00630-8

Acemoglu, D., Chernozhukov, V., Werning, I., \& Whinston, M.D. (2020). Optimal targeted lockdowns in a multi-group SIR model. National Bureau of Economic Research, 27102. https://bit.ly/2N8L0C7

Akpan, I.J., Soopramanien, D., \& Kwak, D.H. (2020a). Cutting-edge technologies for small business and innovation in the era of COVID-19 global health pandemic. Journal of Small Business $\mathcal{E}^{2}$ Entrepreneurship, 1-11. https://doi.org/10.1080/08276331.2020.1799294

Akpan, I.J., Udoh, E.A.P. \& Adebisi, B. (2020b). Small business awareness and adoption of state-ofthe-art technologies in emerging and developing markets, and lessons from the COVID-19 pandemic. Journal of Small Business $\&$ Entrepreneurship, 1-18.

https://doi.org/10.1080/08276331.2020.1820185 
Almeida, F., Santos, J.D., \& Monteiro, J. A. (2020). The challenges and opportunities in the digitalization of companies in a post-COVID-19 World. IEEE Engineering Management Review, 48(3), 97-103. https://doi.org/10.1109/EMR.2020.3013206

Barrero, J.M., Bloom, N., \& Davis, S.J. (2020). COVID-19 is also a reallocation shock. Working paper No. 2020-59, June 25. The Becker Friedman Institute website. https://bit.ly/3nWVcKL

Bartsch, S., Weber, E., Büttgen, M., \& Huber, A. (2021). Leadership matters in crisis-induced digital transformation: how to lead service employees effectively during the COVID-19 pandemic. Journal of Service Management, 32(1), 71-85. https://doi.org/10.1108/JOSM-05-2020-0160

Björklund, T.A., Mikkonen, M., Mattila, P., \& van der Marel, F. (2020). Expanding entrepreneurial solution spaces in times of crisis: Business model experimentation amongst packaged food and beverage ventures. Journal of Business Venturing Insights, 14, e00197.

https://doi.org/10.1016/j.jbvi.2020.e00197

Bond III, E.U., de Jong, A., Eggert, A., Houston, M.B., Kleinaltenkamp, M., Kohli, A.K., Rither, Th., \& Ulaga, W. (2020). The future of B2B customer solutions in a post-COVID-19 economy: managerial issues and an agenda for academic inquiry. Journal of Service Research, 23(4), 401-408. https://doi.org/10.1177/1094670520960230

Bosch-Capblanch X., Lavis, J.N., Lewin, S., Atun, R., Røttingen, J.A., Dröschel D., Beck, L., Abalos, E., El-Jardali, F., Gilson, L., Oliver, S., Wyss, K., Tugwell, P., Kulier, R., Pang, T., \& Haines, A. (2012). Guidance for evidence-informed policies about health systems: rationale for and challenges of guidance development. PLoS Med, 9(3), e1001185.

https://doi.org/10.1371/journal.pmed.1001185

Breier, M., Kallmuenzer, A., Clauss, T., Gast, J., Kraus, S., \& Tiberius, V. (2021). The role of business model innovation in the hospitality industry during the COVID-19 crisis. International Journal of Hospitality Management, 92, 102723. https://doi.org/10.1016/j.ijhm.2020.102723

Campbell, M.C., Inman, J.J., Kirmani, A., \& Price, L.L. (2020). In times of trouble: A framework for understanding consumers' responses to threats. Journal of consumer research, 47(3), 311-326. https://doi.org/10.1093/jcr/ucaa036

Coombs, C. (2020). Will COVID-19 be the tipping point for the intelligent automation of work? A review of the debate and implications for research. International journal of information management, 55, 102182. https://doi.org/10.1016/j.ijinfomgt.2020.102182

Crick, J.M., \& Crick, D. (2020). Coopetition and COVID-19: Collaborative business-to-business marketing strategies in a pandemic crisis. Industrial Marketing Management, 88, 206-213. https:// doi.org/10.1016/j.indmarman.2020.05.016

Dannenberg, P., Fuchs, M., Riedler, T., \& Wiedemann, C. (2020). Digital transition by COVID®19 pandemic? The German food online retail. Tijdschrift voor economische en sociale geografie, 111(3), 543-560. https://doi.org/10.1111/tesg.12453

Di Vaio, A., Boccia, F., Landriani, L., \& Palladino, R. (2020). Artificial intelligence in the agrifood system: Rethinking sustainable business models in the COVID-19 scenario. Sustainability, 12(12), 4851. https://doi.org/10.3390/su12124851

Dwivedi, Y. K., Hughes, D. L., Coombs, C., Constantiou, I., Duan, Y., Edwards, J. S., ... Upadhyay, N. (2020). Impact of COVID-19 pandemic on information management research and practice: Transforming education, work and life. International Journal of Information Management, 55, 102211. http://dx.doi.org/10.1016/j.ijinfomgt.2020.102211

Eggers, F. (2020). Masters of disasters? Challenges and opportunities for SMEs in times of crisis. Journal of Business Research, 116, 199-208. https://doi.org/10.1016/j.jbusres.2020.05.025

Foss, N.J. (2020). The impact of the COVID-19 pandemic on firms' organizational designs. Journal of Management Studies. Online Version of Record before inclusion in an issue. https://doi. org/10.1111/joms.12643

Gamero, H., \& Ostos, J. (2020). Revisión sistemática de literatura sobre factores clave en la identificación de oportunidades de negocio. Retos Revista de Ciencias de la Administración y Economía, 10(20), 307-327. https://doi.org/10.17163/ret.n20.2020.07.

García-Madurga, M.A., \& Esteban-Navarro, M.A. (2020). A project management approach to com- 
petitive intelligence. Journal of Intelligence Studies in Business, 10(3), 8-23.

https://doi.org/10.37380/jisib.v10i3.636

George, G., Lakhani, K.R., \& Puranam, P. (2020). What has changed? The impact of Covid pandemic on the technology and innovation management research agenda. Journal of Management Studies, 57(8), 1754-1758. https://doi.org/10.1111/joms.12634

Grant, M.J. \& Booth, A. (2009). A typology of reviews: an analysis of 14 review types and associated methodologies. Health Information and Libraries Journal, 26(2), 91-108. https://doi.org/10.1111/j.1471-1842.2009.00848.x

Hall, M.C., Prayag, G., Fieger, P., \& Dyason, D. (2021). Beyond panic buying: consumption displacement and COVID-19. Journal of Service Management, 32(1), 113-128. https://doi.org/10.1108/JOSM-05-2020-0151

Hamilton, J. (2020). The strategic change matrix and business sustainability across COVID-19. Sustainability, 12(15), 6026. https://doi.org/10.3390/su12156026

Harris, J.L., Sunley, P., Evenhuis, E., Martin, R., Pike, A., \& Harris, R. (2020). The Covid-19 crisis and manufacturing: How should national and local industrial strategies respond? Local Economy, 35(4), 403-415. https://doi.org/10.1177/0269094220953528

Hartling, L., Guise, J.M., Kato, E., Anderson, J., Belinson, S., Berliner, E., Dryden, D.M., Featherstone, R., Mitchell, M.D., Motu'apuaka, M., noorani, H., Paynter, R., Robinson, K.A., Schoelles, K., Umscheid, C.A., \& Whitlock, E. (2015). A taxonomy of rapid reviews links report types and methods to specific decision-making contexts. Journal of Clinical Epidemiology, 68(12), 1451-1462.e3. https://doi.org/10.1016/j.jclinepi.2015.05.036

Heinonen, K., \& Strandvik, T. (2020). Reframing service innovation: COVID-19 as a catalyst for imposed service innovation. Journal of Service Management, 32(1), 101-112. https://doi.org/10.1108/JOSM-05-2020-0161

Helm, D. (2020). The environmental impacts of the coronavirus. Environmental $\mathcal{E}$ Resource Economics, 76, 21-38. https://doi.org/10.1007/s10640-020-00426-z

Herstatt, C., \& Tiwari, R. (2020). Opportunities of Frugality in the Post-Corona Era. Center for Frugal Innovation Institute for Technology and Innovation Management Hamburg University of Technology. https://bit.ly/2XTYALT

Hite, L.M., \& McDonald, K.S. (2020). Careers after COVID-19: challenges and changes, Human Resource Development International, 23(4), 427-437, https://doi.org/10.1080/13678868.2020.1779576

Hongwei, H., \& Lloyd, H. (2020). The impact of Covid-19 pandemic on corporate social responsibility and marketing philosophy. Journal of Business Research, 116, 176-182. https://doi.org/10.1016/j.jbusres.2020.05.030

Ibarra, D., Bigdeli, A.Z., Igartua, J.I., \& Ganzarain, J. (2020). Business model innovation in established SMEs: A configurational approach. Journal of Open Innovation: Technology, Market, and Complexity, 6(3), 76. https://doi.org/10.3390/joitmc6030076

Kamal, M.M. (2020). The triple-edged sword of COVID-19: understanding the use of digital technologies and the impact of productive, disruptive, and destructive nature of the pandemic. Information Systems Management, 37(4), 310-317. https://doi.org/10.1080/10580530.2020.1820634

Kang, J., Diao, Z., \& Zanini, M. T. (2020). Business-to-business marketing responses to COVID-19 crisis: a business process perspective. Marketing Intelligence $\mathcal{E}$ Planning. Vol. ahead-of-print No. ahead-of-print. https://doi.org/10.1108/MIP-05-2020-0217

Kelly, S.E., Moher, D., \& Clifford, T.J. (2016). Quality of conduct and reporting in rapid reviews: an exploration of compliance with PRISMA and AMSTAR guidelines. Syst Rev 5, 79. https://doi. org/10.1186/s13643-016-0258-9.

Langlois, E.V., Straus, S.E., Mijumbi-Deve, R., Lewin, S., \& Tricco, A.C. (2017). The need for rapid reviews to inform health policy and systems. En A. Tricco, E.V. Langlois, S.E. Strauss (Eds.), Rapid reviews to strengthen health policy and systems: a practical guide (pp. 1-20). Geneva: World Health Organization.

Lau, A. (2020). New technologies used in COVID-19 for business survival: Insights from the Hotel Sector in China. Information Technology \& Tourism, 22(4), 497-504.

https://doi.org/10.1007/s40558-020-00193-z 
Le, K., \& Nguyen, M. (2020). The psychological consequences of COVID-19 lockdowns. International Review of Applied Economics, 1-17. https://doi.org/10.1080/02692171.2020.1853077

Lund, S., Ellingrud, K., Hancock, B., \& Manyika, J. (2020). COVID-19 and Jobs: Monitoring the US Impact on People and Places. McKinsey Global Institute website. https://mck.co/35V6bhE

Manolova, T.S., Brush, C.G., Edelman, L.F., \& Elam, A. (2020). Pivoting to stay the course: How women entrepreneurs take advantage of opportunities created by the COVID-19 pandemic. International Small Business Journal, 38(6), 481-491. https://doi.org/10.1177\%2F0266242620949136

Mele, C., Russo-Spena, T., \& Kaartemo, V. (2020). The impact of coronavirus on business: developing service research agenda for a post-coronavirus world. Journal of Service Theory and Practice. vol. ahead-of-print No. ahead-of-print. https://doi.org/10.1108/JSTP-07-2020-0180

Miles, D., Stedman, M., \& Heald, A. (2020). Living with COVID-19: balancing costs against benefits in the face of the virus. National Institute Economic Review, 253.

https://doi.org/10.1017/nie.2020.30

Moher, D., Liberati, A., Tetzlaff, J., Altman, D.G. \& The PRISMA Group. (2009). Preferred Reporting Items for Systematic Reviews and Meta-Analyses: The PRISMA Statement. BMJ, 339, b2535. https://doi.org/10.1136/bmj.b2535

Møller, M.H., Ioannidis, J.P.A., \& Darmon, M. (2018). Are systematic reviews and meta-analyses still useful research? We are not sure. Intensive Care Medicine, 4, 518-520.

https://doi.org/10.1007/s00134-017-5039-y

Oliver, S., Wilson, M., Melendez-Torres, G.J., Bangpan, M., Dickson, K., \& Vigurs, C. (2017). Selecting rapid review methods for complex questions related to health policy and system improvements. En A. Tricco, E.V. Langlois, S.E. Strauss (Eds.), Rapid reviews to strengthen health policy and systems: a practical guide (pp. 55-70). World Health Organization.

Ortega-Vivanco, M. (2020). Efectos del Covid-19 en el comportamiento del consumidor: Caso Ecuador. Retos Revista de Ciencias de la Administración y Economía, 10(20), 233-247. https://doi.org/10.17163/ret.n20.2020.03.

Paul, J., Menzies, J., Zutshi, A., \& Cai, H. (2020). New and novel business paradigms in and from China and India. European Business Review, 32(5),. 758-800. https://doi.org/10.1108/EBR-092020-0224

Păunescu, C., \& Mátyus, E. (2020). Resilience measures to dealing with the COVID-19 pandemic Evidence from Romanian micro and small enterprises. Management $\mathcal{E}$ Marketing. Challenges for the Knowledge Society, 15(s1), 439-457. https://doi.org/10.2478/mmcks-2020-0026

Pillania, R.K. (2020). COVID-19: A huge opportunity for innovation in marketing. Indian Journal of Marketing, 50, 8-9. http://dx.doi.org/10.17010/ijom\%2F2020\%2Fv50\%2Fi8-9\%2F154693

Plüddemann, A., Aronson, J.K., Onakpoya, I., Heneghan, C., \& Mahtani, K.R. (2018). Redefining rapid reviews: a flexible framework for restricted systematic reviews. BMJ Evidence-Based Medicine, 23(6), pp. 201-203. https://doi.org/10.1136/bmjebm-2018-110990

Putra, I., Sunarsih, N., Novitasari, L., \& Setini, M. (2020). Exploring the relationship between social capital, innovation capability and innovation during the coronavirus pandemic. Uncertain Supply Chain Management, 8(4), 857-864. http://dx.doi.org/10.5267/j.uscm.2020.5.007

Rapaccini, M., Saccani, N., Kowalkowski, C., Paiola, M., \& Adrodegari, F. (2020). Navigating disruptive crises through service-led growth: The impact of COVID-19 on Italian manufacturing firms. Industrial Marketing Management, 88, 225-237.

https://doi.org/10.1016/j.indmarman.2020.05.017

Ratten, V. (2020). Coronavirus and international business: An entrepreneurial ecosystem perspective. Thunderbird International Business Review, 62(5), 629-634. https://doi.org/10.1002/tie.22161

Schaltegger, S. (2020). Sustainability learnings from the COVID-19 crisis. Opportunities for resilient industry and business development. Sustainability Accounting, Management and Policy Journal. Vol. ahead-of-print No. ahead-of-print. https://doi.org/10.1108/SAMPJ-08-2020-0296

Seddon, P.B., Lewis, G.P., Freeman, P., \& Shanks, G. (2004). The case for viewing business models as abstractions of strategy. Communications of the association for Information Systems, 13(25), 427-442. https://doi.org/10.17705/1CAIS.01325 
Seetharaman, P. (2020). Business models shifts: Impact of Covid-19. International Journal of Information Management, 54, 102173. https://doi.org/10.1016/j.ijinfomgt.2020.102173

Shankar, K. (2020). The impact of COVID-19 on IT services industry-expected transformations. British Journal of Management, 31(3), pp. 450-452. https://doi.org/10.1111/1467-8551.12423

Sharma, P., Leung, T.Y., Kingshott, R.P., Davcik, N.S., \& Cardinali, S. (2020). Managing uncertainty during a global pandemic: An international business perspective. Journal of Business Research, 116, 188-192. https://dx.doi.org/10.1016\%2Fj.jbusres.2020.05.026

Sheth, J. (2020a). Impact of Covid-19 on Consumer Behavior: Will the Old Habits Return or Die? Journal of Business Research, 117, 280-283. https://doi.org/10.1016/j.jbusres.2020.05.059

Sheth, J. (2020b). Business of business is more than business: Managing during the Covid crisis. Industrial Marketing Management, 88, 261-264. https://doi.org/10.1016/j.indmarman.2020.05.028

Sigala, M. (2020). Tourism and COVID-19: impacts and implications for advancing and resetting industry and research. Journal of Business Research, 117, 312-321. https://doi.org/10.1016/j.jbusres.2020.06.015

Sucharew, H., \& Macaluso, M. (2019). Methods for Research Evidence Synthesis: The Scoping Review Approach. Journal of Hospital Medicine 14, 416-418. https://doi.org/10.12788/jhm.3248

Teece, D., Peteraf, M., \& Leih, S. (2016). Dynamic Capabilities and Organizational Agility: Risk, Uncertainty, and Strategy in the Innovation Economy. California Management Review, 58(4), 13 35. https://doi.org/10.1525\%2Fcmr.2016.58.4.13

Tisdell, C.A. (2017). Information technology's impacts on productivity and welfare: a review. International Journal of Social Economics, 44(3), 400-413. http://dx.doi.org/10.1108/IJSE-06-2015-0151

Tisdell, C.A. (2020). Economic, social and political issues raised by the COVID-19 pandemic. Economic analysis and policy, 68, 17-28. https://doi.org/10.1016/j.eap.2020.08.002

Tricco, A.C., Antony, J., Zarin, W., Strifler, L., Ghassemi, M., Ivory, J., Perrier, L., Hutton, B., Moher, D., \& Straus, S.E. (2015). A scoping review of rapid review methods. BMC Medicine, 13(224). https://bit.ly/2ZT1PUN

Tricco, A.C., Soobiah, C., Antony, J., Cogo, E., MacDonald, H., Lillie, ... \& Kastner, M. (2016). A scoping review identifies multiple emerging knowledge synthesis methods, but few studies operationalize the method. Journal of Clinical Epidemiology 73, 19-28.

https://doi.org/10.1016/j.jclinepi.2015.08.030.

Tricco, A.C., Zarin, W., Nincic, V., Rios, P., Khan, P.A., Ghassemi, M., Motiwala, S., Pham, Ba',, Oliver, S., Straus, S.E., \& Langlois, E.V. (2017). Engaging policymakers and health systems managers in the conduct of rapid reviews. En A. Tricco, E.V. Langlois, S.E. Strauss (Eds.), Rapid reviews to strengthen health policy and systems: a practical guide (pp. 71-82). World Health Organization. https://bit.ly/3pYalg3

Villasmil-Espinoza, J.J. (2021). Implicaciones de la inteligencia artificial para la humanidad. Revista de la Universidad del Zulia,12(32), 4-6. https://doi.org/10.46925//rdluz.32.01.

Watt, A., Cameron, A., Sturm, L., Lathlean, T., Babidge, W., Blamey, S., Facey, K., Hailey, D., Norderhaug, I., \& Maddern, G. (2008). Rapid reviews versus full systematic reviews: an inventory of current methods and practice in health technology assessment. Int J Technol Assess Health Care, 24(2), 133-139. https://10.1017/S0266462308080185.

Williams, T.A., Gruber, D.A., Sutcliffe, K.M., Shepherd, D.A., \& Zhao, E.Y. (2017). Organizational response to adversity: Fusing crisis management and resilience research streams. Academy of Management Annals, 11(2), 733-769. https://psycnet.apa.org/doi/10.5465/annals.2015.0134

Zhu, X., \& Liu, K. (2020). A systematic review and future directions of the sharing economy: business models, operational insights and environment-based utilities. Journal of Cleaner Production, 125209. In press. https://doi.org/10.1016/j.jclepro.2020.125209 